\title{
Upstream shear-layer stabilisation via self-oscillating trailing edge flaplets
}

\author{
Edward Talboys $^{1}\left[\right.$ (1) Christoph Brücker $^{1}$
}

Received: 23 March 2018 / Revised: 14 August 2018 / Accepted: 18 August 2018 / Published online: 4 September 2018

(c) The Author(s) 2018

\begin{abstract}
The flow around a symmetric aerofoil (NACA 0012) with an array of flexible flaplets attached to the trailing edge has been investigated at Reynolds numbers of 100,000-150,000 using high-speed time-resolved particle image velocimetry (HS TRPIV) and motion tracking of the flaplets' tips. Particular attention has been made on the upstream effect on the boundary layer evolution along the suction side of the wing, at angles of attack of $0^{\circ}$ and $10^{\circ}$. For the plain aerofoil, without flaplets, the boundary layer on the second half of the aerofoil shows the formation of rollers as the shear-layer rolls-up in the fundamental instability mode (linear state). Proper orthogonal decomposition analysis shows that non-linear modes are also present, the most dominant being the pairing of successive rollers. When the flaplets are attached, it is shown that the flow-induced oscillations of the flaplets are able to create a lock-in effect that stabilises the linear state of the shear layer, whilst delaying or damping the growth of non-linear modes. It is hypothesised that the modified trailing edge is beneficial for reducing drag and can reduce aeroacoustic noise production in the lower frequency band, as indicated by an initial acoustic investigation.
\end{abstract}

\section{List of symbols}

$U_{\infty} \quad$ Free-stream velocity $(\mathrm{m} / \mathrm{s})$

$U_{0} \quad$ Convective velocity $(\mathrm{m} / \mathrm{s})$

$U_{\text {es }} \quad$ Boundary layer edge velocity $(\mathrm{m} / \mathrm{s})$

$u^{\prime} \quad$ Streamwise velocity fluctuations $(\mathrm{m} / \mathrm{s})$

$v^{\prime} \quad$ Wall-normal velocity fluctuations $(\mathrm{m} / \mathrm{s})$

$c \quad$ Aerofoil chord (m)

$R e_{\mathrm{c}} \quad$ Chord-based Reynolds Number

$\alpha \quad$ Angle of attack $\left({ }^{\circ}\right)$

$f_{0} \quad$ Shear-layer fundamental frequency $(\mathrm{Hz})$

$f_{0-1 / 2} \quad$ Shear-layer instability frequency $(\mathrm{Hz})$

$f_{\mathrm{k}} \quad$ Vibration frequency at $k$ th mode $(\mathrm{Hz})$

$\alpha_{\mathrm{k}} \quad$ Wave-number at the $k$ th vibration mode

$\delta^{*} \quad$ Boundary layer displacement thickness (m)

$\lambda_{0} \quad$ Shear-layer roll-up wavelength (m)

$S t_{0}^{*} \quad$ Shear-layer Strouhal Number

$s \quad$ Spanwise width of flaplet $(\mathrm{m})$

$L \quad$ Length of flaplet (m)

$d \quad$ Spacing between flaplet (m)

$h \quad$ Thickness of flaplet (m)

w $\quad$ Flaplet vertical displacement (m)

$D \quad$ Damping coefficient $(\mathrm{kg} / \mathrm{s})$

Edward Talboys

Edward.Talboys.1@city.ac.uk

1 City, University of London, Northampton Square, London EC1V 0HB, UK

$\begin{array}{ll}\zeta & \text { Damping ratio } \\ \omega_{\mathrm{n}} & \text { Natural frequency }(\mathrm{rad} / \mathrm{s}) \\ \omega_{\mathrm{d}} & \text { Damped frequency }(\mathrm{rad} / \mathrm{s}) \\ \phi & \text { Phase delay }(\mathrm{rad}) \\ E & \text { Elastic modulus }\left(\mathrm{N} / \mathrm{m}^{2}\right) \\ I & \text { Area moment of inertia }\left(\mathrm{m}^{4}\right) \\ \rho_{\mathrm{f}} & \text { Flaplet material density }\left(\mathrm{kg} / \mathrm{m}^{3}\right) \\ K & \text { Non-dimensional bending stiffness }\end{array}$

\section{Introduction}

In recent years extensive research has been carried out by turning to nature for inspiring new designs in man-made aircraft and improving aerodynamic performance or reducing emissions such as noise or pollution. Ever increasing air travel and human habitation near airports, has led to the urgent need for cleaner and quieter air travel. A few solutions which have been previously investigated are: leading edge undulations (Miklosovic et al. 2004), serrated trailing edges (Howe 1991), slitted trailing edges (Gruber et al. 2010), brush-like edge extensions (Herr and Dobrzynski 2005) and porous aerofoils (Geyer et al. 2010). In the present study, a flexible trailing edge consisting of an array of small elastic flaplets, mimicking the tips of bird feathers, is used.

The specific arrangement and structure of feathers on the trailing edge of an owl's wing is well known to be one of 
the key mechanisms that the bird uses to enhance noise suppression (Jaworski and Peake 2013). In addition, secondary feathers on the upper side of the wings of the steppe eagle (Aquila nipalensis) (Carruthers et al. 2007) and the peregrine falcon (Falco peregrinus) (Ponitz et al. 2014), to give a few examples, have been observed to pop-up as the birds attack prey or come into land at a high angle of attack. This phenomenon has been the subject of many research studies.

Schlüter (2010) could show that by attaching rigid flaps, via a hinge, on the upper surface of the wing, the $C_{\mathrm{L}} \max$ is increased for a series of tested aerofoils (NACA 0012, NACA 4412, SD 8020). Schlüter also showed that the flaps bring the additional benefit of gradual stall rather than a more severe lift crisis. Osterberg and Albertani (2017) carried out a similar study on a flat plate subjected to high angles of attack, coming to the same conclusion.

Brücker and Weidner (2014) used flexible flaplets attached on the suction side of a NACA 0020 aerofoil that was subjected to a ramp-up motion. The results show a considerable delay in dynamic stall. Flap length and flap chordwise spacing were varied and it was found that the most successful configuration was two rows of flaps of length 0.1 c spaced $0.15-0.2 \mathrm{c}$ in the chordwise direction. The wavelength of the rollers in the shear layer was found to be of the same order, $0.15-0.2 \mathrm{c}$. This led to the conclusion that the flaplets resulted in a lock-in effect with these rollers, as the two spacial length scales were comparable. Concluding that this lock-in effect stabilises the shear layer. Rosti et al. (2017) then built on these findings of by carrying out a DNS (direct numerical simulation) parametric study. The flap element was rigid but coupled to the aerofoil surface by a torsional spring type coupling. It was found that when the flap oscillations were at the same frequency as the shear-layer roll-up, the mean lift coefficient was at its highest.

Similar effects, as observed for aerofoils, have also be seen on tests with bluff bodies. A series of studies on cylinder flows with flaplets attached on the aft half of the cylinder have been carried out by Kunze and Brücker (2012), Kamps et al. (2016) and Geyer et al. (2017). Kunze and Brücker (2012) saw that the presence of the flexible elements allowed the shedding frequency to be locked in with the most dominant eigen-frequency of the flaplets. This hints to a similar lock-in effect of the shear-layer roll-up observed for the aerofoils (Rosti et al. 2017). Consequently, the flow structure in the wake is changed and a reduced wake deficit is observed. Kamps et al. (2016) then tested the same cylinder/flaplet configuration in an aeroacoustic wind tunnel and could show that the flaplets overall reduce noise, both in the tonal and the broadband components.

Talboys et al. (2018) have very recently, during the review process of the current study, carried out an aeroacoustic campaign with the present configuration of aerofoil and self-oscillating trailing edge flaplets. They have found that when the flaplets are attached to the trailing edge, an acoustic reduction in the low-to-medium frequency range $(100 \mathrm{~Hz}-1 \mathrm{kHz})$ is observed over a large range of Reynolds Numbers and angles of attack. This supports the findings of the preliminary investigation carried out by Kamps et al. (2017) on a NACA 0010 with flexible flaplets attached to the trailing edge. In another recent publication Jodin et al. (2018) uses an active control technique with a vibrating solid trailing edge, which can oscillate up to $400 \mathrm{~Hz}$ with a peak deflection in the order of $1 \mathrm{~mm}$. By using high-speed PIV in the wake it was seen that when the trailing edge oscillations are actuated, the wake thickness was reduced by as much as $10 \%$ and accompanying lift force measurements showed a $2 \%$ increase.

The present study builds off these previous studies in order to study the benefit/impact of flexible flaplets being attached to the trailing edge rather than on the aerofoil body. Tests of such modifications of the trailing edge by Kamps et al. (2017) and Talboys et al. (2018) already show promising results in noise reduction. However, no details of the flow-structure and the fluid-structure interaction are known which might explain the observed aeroacoustical modification. This is the motivation and purpose of the present study.

\section{Experimental setup}

The experiments were carried out in the Handley Page laboratory at City, University of London in a closed loop wind tunnel. The test section of the tunnel is $0.81 \mathrm{~m}$ by $1.22 \mathrm{~m}$ in cross-section, and has a turbulence intensity of $0.8 \%$. A NACA 0012 aerofoil, with a chord of $0.2 \mathrm{~m}$ and span of $0.52 \mathrm{~m}$, was used for the present study. One side of the aerofoil spanned to the floor of the tunnel and an endplate was affixed to the exposed end to negate any end effects. The aerofoil was 3D printed in two sections with a small perspex section in the measurement window, as per Fig. $1 \mathrm{~b}$. The use of perspex in this region improves the quality of the PIV recordings close to the surface. Air flow with density of $\rho=1.2 \mathrm{~kg} / \mathrm{m}^{3}$ was applied at different flow speeds $U_{\infty}$. Three chord-based Reynolds numbers were analysed in this study: 100,000, 125,000 and 150,000; at two different angles of attack: $0^{\circ}$ and $10^{\circ}$. A $0.3 \mathrm{~mm}$ thick boundary layer trip was implemented at $0.2 \mathrm{c}$ on both sides of the aerofoil to ensure that the boundary layer (BL) was turbulent.

The trailing edge with the flexible flaplets was made from a polyester foil, of thickness $h=180 \mu \mathrm{m}$, which was laser-cut at one long side to form an array of individual uniform flaplets. Each rectangular flaplet has on the long side a length of $L=20 \mathrm{~mm}$ and on the short side a width of $s=5 \mathrm{~mm}$ with a gap of $d=1 \mathrm{~mm}$ in spanwise direction (see Fig. 1b). The foil was adhered to the pressure side of the aerofoil using thin double-sided tape such that the flaplets 
face downstream, with their free end located at a distance of $x / c=1.1 \mathrm{c}$ downstream of the trailing edge. The flaplets form a mechanical system of a one-sided clamped rectangular cantilever beam which is free to oscillate perpendicular to the mean-flow direction.

\subsection{Velocity field measurements}

High-speed time-resolved particle image velocimetry (HS TR-PIV) measurements were carried out using a $2 \mathrm{~mm}$ thick double-pulsed Nd:YLF laser sheet in a standard planar setup. A high-speed camera (Phantom Miro M310, window size $1280 \times 800$ pixels) equipped with a macro-lens, Tokina $100 \mathrm{~mm}$, with $\mathrm{f} / 8$ was used in frame straddling mode. Olive oil seeding particles, of approximate size $1 \mu \mathrm{m}$, were added to the flow downstream of the model. A number of 500 pairs of images were captured at a frequency of $1500 \mathrm{~Hz}$ with the pulse separation time being altered for each case, given in Table 1.

The raw images were then processed using the TSI Insight $4 \mathrm{G}$ software which uses the method of $2 \mathrm{D}$ cross correlation. The first pass interrogation window size was $32 \times 32$ pixels, with a $50 \%$ overlap. The size was then reduced to $16 \times 16$ pixels for the subsequent pass. A $3 \times 3$ median filter was then applied to validate the local vectors, any missing or spurious vectors were interpolated using the local mean.

Due to the limited pixel resolution of the high-speed camera and the requirement to have high-speed TR-PIV with good spatial resolution, only a small portion of the flow field could be investigated. Therefore in the present study the region of focus was the aft half and trailing edge sections of the aerofoil, as seen in Fig. 1b. This region was selected as this is where the modification of the flow, by the flaplets, is thought to be most prevalent.

\subsection{Flap motion tracking}

To track and record the real time motion of the flaplets with high resolution, a high power LED (HARDsoft IL-106G) was used alongside a second high-speed camera (Phantom Miro M310) with a Nikon $50 \mathrm{~mm}$ f/1.8 lens. The LED was directed at the flaplets and the back reflections were seen on a translucent screen adhered to the transparent wind tunnel side wall (see Fig. 1a). Due to the optical lever-arm condition, small deflections of the flaplets led to a large displacement of the back-scattered light on the screen. The recordings were taken at $3200 \mathrm{~Hz}$, with an exposure time of $320 \mu \mathrm{s}$ and an aperture of $\mathrm{f} / 1.8$. In total $2.5 \mathrm{~s}$ of motion was recorded on the Phantom Camera Control Application (PCC), resulting in 8000 images for each case. An edge detection code (Matlab) was then used to track the flap tips motion over time. Spurious data points were removed from the data set and subsequently interpolated prior to a lowpass filter (4th order Butterworth low-pass with a cut-off frequency of $600 \mathrm{~Hz}$ ) being applied to the data.

\section{Results}

The test cases mentioned in Sect. 2, were run both with and without the flaplets in order to ascertain a baseline for comparative analysis. For the remainder of the report, the

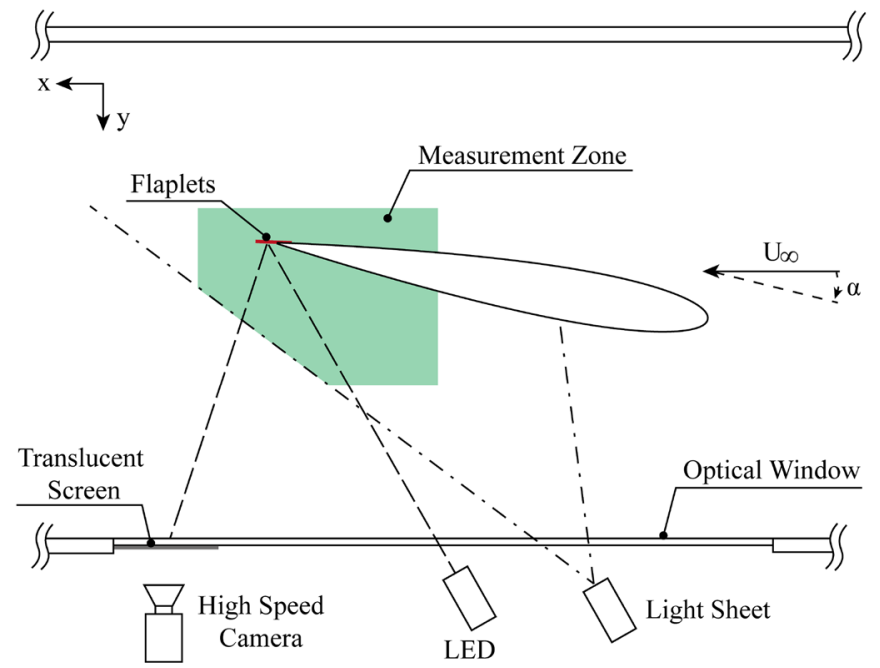

(a) Plan View

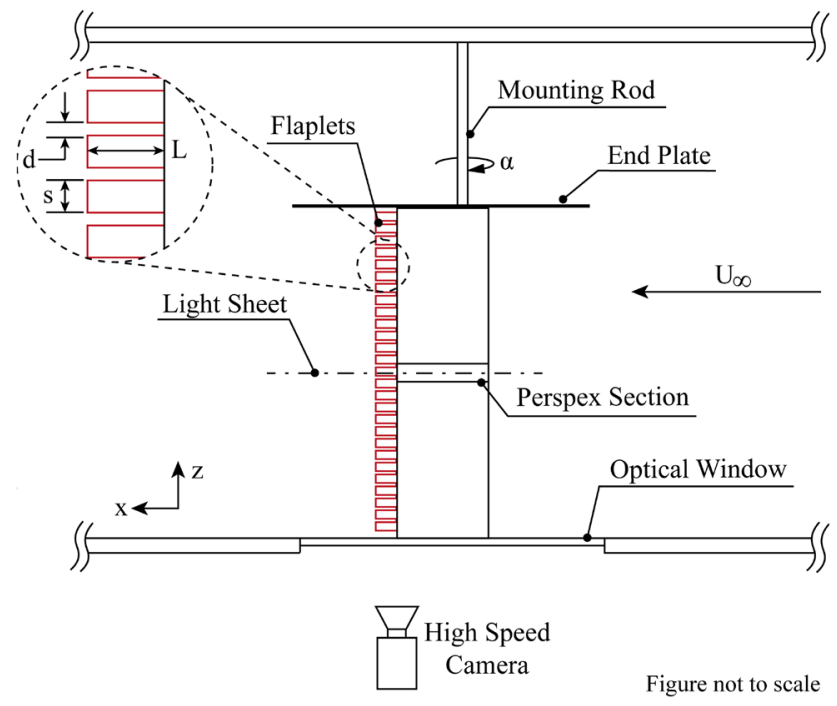

(b) Side View

Fig. 1 Experimental setup 
Table 1 TR-PIV parameters used for the two different $R e_{c}$

\begin{tabular}{llll}
\hline$R e_{\mathrm{c}}$ & Aperture size & $\begin{array}{l}\text { Pulse separation } \\
(\mu \mathrm{s})\end{array}$ & $\begin{array}{l}\text { Capture } \\
\text { frequency } \\
(\mathrm{Hz})\end{array}$ \\
\hline 100,000 & $\mathrm{f} / 8$ & 80 & 1500 \\
150,000 & $\mathrm{f} / 8$ & 30 & 1500 \\
\hline
\end{tabular}

nomenclature in Table 2 will be used and only results for $R e_{\mathrm{c}}=100,000$ and $R e_{\mathrm{c}}=150,000$ are shown.

\subsection{Flaplet response to step input}

As previously mentioned (Sect. 2.2), the flaplets can be understood as thin rectangular cantilever beams, clamped at one side and free to oscillate in their natural bending mode in the flow environment. The subsequent equation of motion from bending beam theory leads to the following ODE of the mechanical system (Stanek 1965):

$\mathrm{EI} \frac{\partial^{2} w}{\partial x^{4}}=-\rho_{\mathrm{f}} A \frac{\partial^{2} w}{\partial t^{2}}-\frac{D}{L} \frac{\partial w}{\partial t}$

The general solution for a damped harmonic oscillators is then:

$\hat{w}(t)=\frac{w}{w_{0}} e^{-\zeta \omega_{\mathrm{n}} t} \cos \left(\omega_{\mathrm{d}} t-\phi\right)$

In the case that the system is only weakly damped, the natural frequency results to:

$f_{\mathrm{k}}=\frac{\alpha_{\mathrm{k}}^{2} h}{2 \pi L^{2}} \sqrt{\frac{\mathrm{EI}}{12 \cdot \rho_{\mathrm{f}}}}$

A step-response test was conducted with a single flaplet being bent out of the equilibrium to an amplitude of $w_{0}$, and subsequently unloaded, after which the flaplet oscillates back to its equilibrium position at rest. The tip motion was recorded by a high-speed camera (Phantom Miro M310, $1200 \times 800$ pixels at $3200 \mathrm{~Hz}$ ) and the previously mentioned edge detection script was used to track the tip. The recorded response, Fig. 2, gives a $Q$-factor of $61.6\left(Q_{\text {factor }}=1 / 2 \zeta\right)$, which indicates a very weakly damped harmonic oscillator. The natural frequency of the flaplets is $107 \mathrm{~Hz}$ as obtained by analysing the spectrum of the signal. With the known solution of Eq. 3, for the weakly damped harmonic oscillator, and using the first natural bending mode of the beam, $\alpha_{1}=1.875$, one can estimate the Young's modulus of the flap material. Equation 3 has been further used to evaluate at which point the flaplets will go into the second vibration mode, for this case $\alpha_{2}=4.694$ leading to $f_{2}=671 \mathrm{~Hz}$ (Table 3).
Table 2 Test matrix nomenclature

\begin{tabular}{llll}
\hline Test name & $R e_{\mathrm{c}}$ & $\alpha$ & Trailing edge \\
\hline $100-0-\mathrm{P}$ & 100,000 & $0^{\circ}$ & Plain \\
$100-0-\mathrm{F}$ & 100,000 & $0^{\circ}$ & Flaplets \\
$100-10-\mathrm{P}$ & 100,000 & $10^{\circ}$ & Plain \\
$100-10-\mathrm{F}$ & 100,000 & $10^{\circ}$ & Flaplets \\
$150-0-\mathrm{P}$ & 150,000 & $0^{\circ}$ & Plain \\
$150-0-\mathrm{F}$ & 150,000 & $0^{\circ}$ & Flaplets \\
$150-10-\mathrm{P}$ & 150,000 & $10^{\circ}$ & Plain \\
$150-10-\mathrm{F}$ & 150,000 & $10^{\circ}$ & Flaplets \\
\hline
\end{tabular}

The non-dimensional bending stiffness of the flaplets has been calculated from Eq. 4 giving a minimum value of $K=6.13 \times 10^{-3}$ at $R e_{\mathrm{c}}=150,000$ and a maximum, $K=13.91 \times 10^{-3}$ at $R e_{\mathrm{c}}=100,000$. Overall, it is concluded that the flaplets are of sufficient flexibility in order to easily react with pressure fluctuations in the present flow conditions.

$K=\frac{\mathrm{EI}}{\rho L^{3} U_{\infty}^{2}}=\frac{\mathrm{Es}}{12}\left(\frac{h}{L}\right)^{3} \frac{1}{\rho U_{\infty}^{2}}$

\subsection{Flow field}

The high-speed PIV measurements were analysed to gather the coherent structures in the shear layer by conditional averaging and POD analysis. Therefore, a virtual probe location has been selected within the PIV field, which acts to give an indicative value for the passage of rollers. It is found that the proper location in the field is where the maximum RMS streamwise velocity fluctuations ( $u^{\prime}$ RMS) occur. The wall-normal position of the maximum $u^{\prime}$ RMS gives an approximate boundary layer displacement thickness $\left(\delta^{*}\right)$ and the position at which the inflection in the boundary layer occurs. Hence this location corresponds to the wall-normal coordinate where the shear-layer instability is most prevalent (Dovgal et al. 1994). For the cases 150-10-P and 150-10-F, $\delta^{*} / \mathrm{c}$ was found to be 0.027 and 0.028 , respectively, at the chordwise location $x / c=0.85$. Accordingly, the probe location has been set at $x / c=0.85$ and $y / c=\delta^{*} / \mathrm{c}$.

The fluctuating vertical velocity $\left(v^{\prime}\right)$ signal was recorded at the probe location (average of the $3 \times 3$ neighbouring vectors) and it was observed, from the PIV results, that the presence of a shear layer roller travelling through the probe area corresponded to a peak in the $v^{\prime}$ component. Therefore in order to visualise a clear depiction of these structures passing through the probe area, the velocity fields were conditionally averaged with the peaks in $v^{\prime}$. In order to observe how the structures develop in time, 5 frames preceding and 


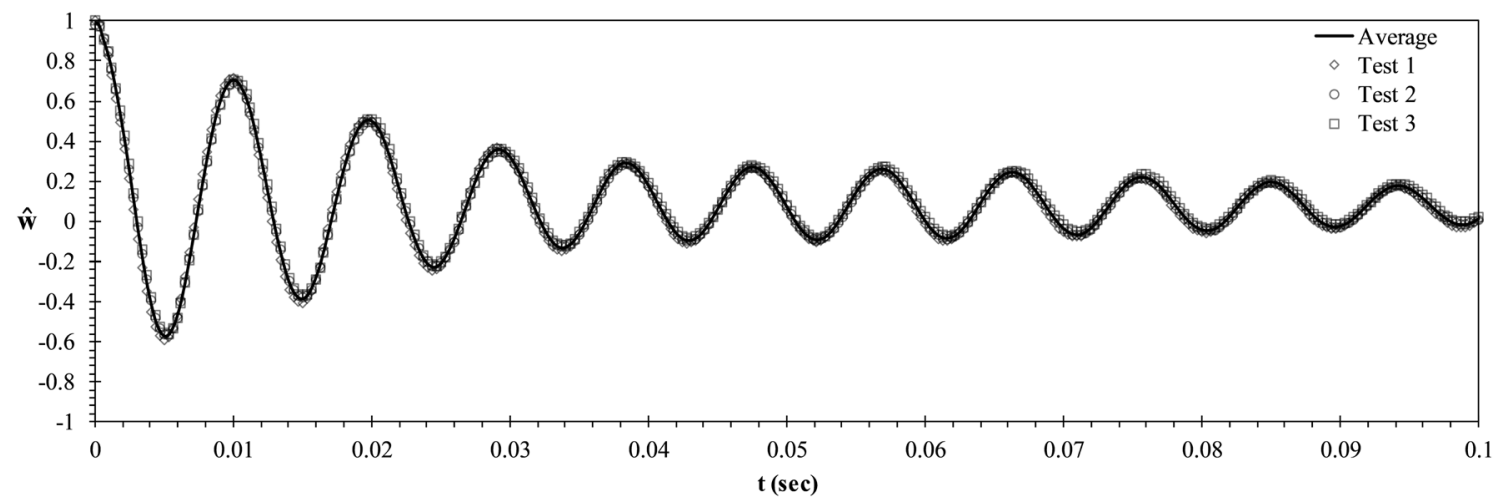

Fig. 2 Response of a singular flaplet to a step input

Table 3 Flaplet dynamics and material properties

\begin{tabular}{ll}
\hline Property & Value \\
\hline$f_{1}$ at $\alpha_{1}$ & $107 \mathrm{~Hz}$ \\
$f_{2}$ at $\alpha_{2}$ & $671 \mathrm{~Hz}$ \\
$\rho_{\mathrm{f}}$ & $1440 \mathrm{~kg} / \mathrm{m}^{3}$ \\
$E$ & $3.12 \mathrm{GPa}$ \\
\hline
\end{tabular}

following were also stored and averaged. From these averaged frames the Q-criterion was subsequently computed and the convection speed was calculated; see Fig. 3. All cases fell in the range $U_{0} / U_{\text {infty }}=0.45-0.55$. A further breakdown of the individual cases can be seen in Table 4 .

In order to select the dominant modes in the flow field, a proper orthogonal decomposition (POD) of the fluctuating velocity field was carried out. POD reduces the field into modes, whereby each mode is a certain dominant flow feature or structure. Mode 1 represents the distribution of the Reynolds stresses in the boundary layer and is not shown here. In the present case the dominant features are the observed rollers that originate from the instability of the shear layer and the roll-up into vortices as seen in Fig. 3. The spatial reconstruction of two of the dominant modes can be seen in Fig. 4. Mode 4 (Fig. 4a) is the mode which corresponds to the fundamental instability, leading to the shear-layer rolling up into regular rows of spanwise rollers. A proof of being it mode 4 is given later based on previous research of boundary layers on aerofoils. Then, mode 2 , Fig. 4b, corresponds to half of the fundamental instability which indicates the presence of a non-linear state in the shear layer. This mode occurs at half the wavelength of the fundamental mode and represents a subharmonic feature generated by pairing of successive rollers. Mode 3 is a mixture of mode 2 and mode 4 and is linked with the fundamental mode with a factor of $2 / 3$. Thus it seems to be an intermediate state.
In order to justify our selection of the mode 4 representing the fundamental shear-layer instability, the Strouhal number of the mode was calculated and compared to previous research on similar aerofoils, see Yarusevych et al. (2009). They suggested a certain range of the Strouhal number exists in which the fundamental mode should fall in when scaling the frequency $\left(f_{0}\right)$ with the wavelength of the fundamental roll-up $\left(\lambda_{0}\right)$ and the boundary layer edge velocity $\left(U_{\mathrm{es}}\right)$, see Eq. 5. Analytically Yarusevych et al. (2009) showed that this quantity should be in the region of $0.45 \leq S t_{0}^{*} \leq 0.5$ and more recently this range was increased to $0.3 \leq S t_{0}^{*} \leq 0.5$ by Boutilier and Yarusevych (2012). Values for $\lambda_{0}$ were obtained from the POD (Fig. 4) and the convection velocity of the vortex cores were calculated from the conditional averaging (Fig. 3). The values of $S t_{0}^{*}$ obtained in the present study are within the limits set by Boutilier and Yarusevych (2012) and are in agreement also with previous studies (Boutilier and Yarusevych 2012; Thomareis and Papadakis 2017; Yarusevych et al. 2009; Brücker and Weidner 2014). Similar roller wavelengths were observed by Brücker and Weidner (2014), where the wavelength they found was $0.15-0.2 \mathrm{c}$. The value of the fundamental frequency could then be back calculated from this relationship and is presented in Table 4 as $f_{0 \mathrm{c}}$. These calculated values show good agreement with the values obtained from the $v^{\prime}$ spectra at the PIV probe location (Fig. 5), giving confidence in the presented frequencies. Additional analysis is carried out on the temporal coefficients of the POD modes as they represent the temporal signature of the mode (Semeraro et al. 2012; Meyer et al. 2007). This helps to link the corresponding mode 4 with $f_{0}$ (Fig.5c-f) and mode 2 with $f_{0-1 / 2}$ (Fig. 5b-e), as the identified frequencies in the $v^{\prime}$ spectra (Fig. 5a-d).

$S t_{0}^{*}=\frac{f_{0} \lambda_{0}}{U_{\mathrm{es}}}=\frac{U_{0}}{U_{\mathrm{es}}}$ 

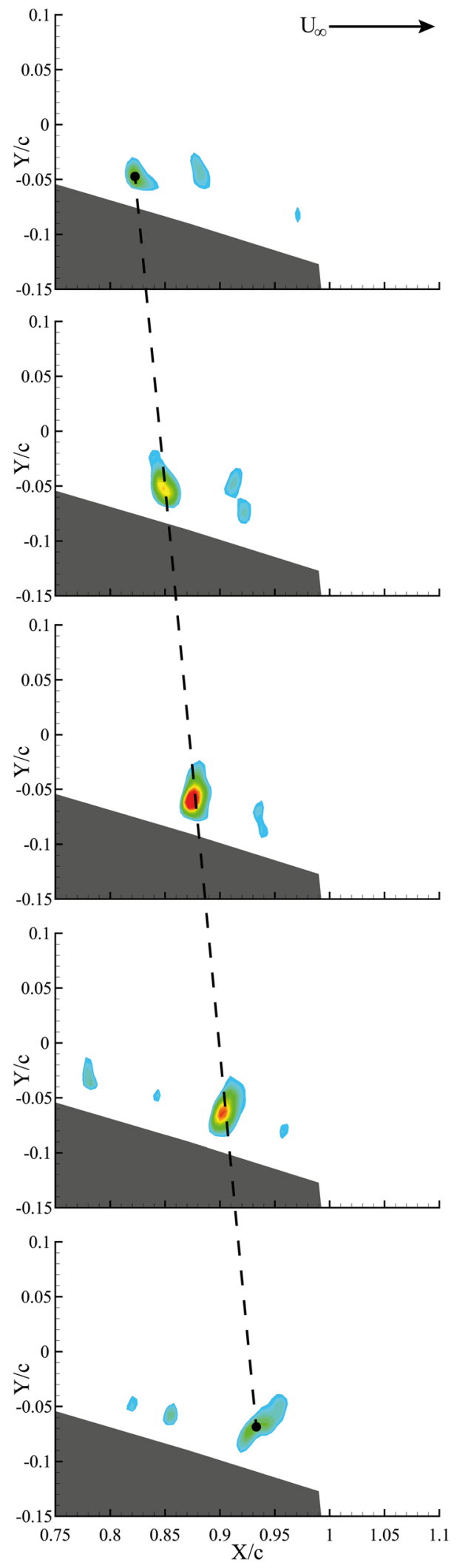

Fig. 3 Q-criterion for case 100-10-F. Time spacing in between each image is: $\Delta t^{*}=\Delta t \cdot U_{\infty} / c=0.055$
In Fig. 5a, 150-10-P, the dominant peak is observed at $f_{0-1 / 2}$, which corresponds to approximately half of the fundamental frequency and indicates the presence of mode 2 . Therefore, at this condition, the shear layer upstream and near the trailing edge is already in a non-linear state with pairing of the rollers happening more often. This pairing effect has been seen in many previous studies of planar shear flows (Ho and Huang 1982; Rajagopalan and Antonia 2005; Rodríguez et al. 2013; Perret 2009). It should be noted that this frequency herein is not exactly half of the fundamental peak, which is reasonable as the position of the transition point of the fundamental instability is typically fluctuating in the shear layer; hence this gives a more 'broadband' region where this frequency is seen (Prasad and Williamson 1997; Rajagopalan and Antonia 2005; Dong et al. 2006; Yarusevych et al. 2009).

Once the flaplets are attached to the aerofoil and the flow is studied again at the same conditions (Fig. 5d). The $f_{0-1 / 2}$ peak is obviously suppressed and the $f_{0}$ is now the dominant frequency. Meanwhile, as shown further below, the flaplets oscillate at their natural frequency with an average amplitude of about $0.4 \mathrm{~mm}(0.2 \% \mathrm{c})$, excited by the observed rollers over the trailing edge. The shift of the dominant peak back to the fundamental instability indicates that the shear layer has now been stabilised and the growth of non-linear modes has been damped, thus reducing the tendency of vortex pairing. This stabilisation is thought to be caused by a lock-in effect between the oscillating flaplets and the shear-layer fundamental instability, once the frequency of the latter exceeds the natural frequency of the flaplets. Such that they start to oscillate in the flow. This conclusion is based upon a similar observation of flow stabilisation with flaplets attached to the aft part of an aerofoil and a bluff body (Brücker and Weidner 2014; Rosti et al. 2017; Kunze and Brücker 2012), where the flaps act as 'pacemaker' and alter the shedding cycle, leading to a reduction in drag and lift fluctuations. It is worth to note that the herein observed stabilisation goes together with a slight reduction in the thickness of the local boundary layer edge $(\delta)$. Table 4 shows that with the flaplets the thickness has been reduced by $7.5 \%$, inferring that with the attached flaplets the aerodynamic performance of the aerofoil might also benefit. It is hypothesised that the reduced thickness is the consequence of less pairing events in the stabilised situation, because the pairing causes a strong wall-normal momentum exchange and therefore a thickening of the boundary layer.

In order to investigate the chordwise sensitivity of the spectra, an additional 15 points both upstream and downstream of the presented probe location were averaged. The additional locations were taken at the same wall-normal position $\left(\delta^{*} / c\right)$ and with a chordwise spacing of $0.5 \% \mathrm{c}$. The 
Table 4 Spectra results, Strouhal number and boundary layer quantities

\begin{tabular}{|c|c|c|c|c|c|c|c|c|c|c|c|}
\hline \multirow[t]{2}{*}{ Test cases } & \multicolumn{2}{|c|}{ PIV probe } & \multicolumn{2}{|l|}{ POD } & \multicolumn{5}{|c|}{ Shear-layer Strouhal number parameters } & \multicolumn{2}{|c|}{ Boundary layer } \\
\hline & $f_{0} c / U_{\infty}$ & $f_{0-1 / 2} c / U_{\infty}$ & $f_{0} c / U_{\infty}$ & $f_{0-1 / 2} c / U_{\infty}$ & $U_{0} / U_{\infty}$ & $\lambda_{0} / c$ & $U_{\mathrm{es}} / U_{\infty}$ & $S t_{0}^{*}$ & $f_{0 \mathrm{c}} c / U_{\infty}$ & $\delta / c$ & $\delta * \mathrm{c}$ \\
\hline $100-10-\mathrm{P}$ & 4 & - & 4 & - & 0.466 & 0.138 & 1.02 & 0.456 & 3.36 & 0.0754 & 0.0234 \\
\hline $100-10-\mathrm{F}$ & 3.52 & - & 3.52 & - & 0.498 & 0.154 & 1.02 & 0.488 & 3.23 & 0.0701 & 0.0234 \\
\hline $150-10-\mathrm{P}$ & 4.14 & 2.76 & 4.14 & 2.55 & 0.497 & 0.125 & 1.04 & 0.478 & 3.98 & 0.0652 & 0.0285 \\
\hline $150-10-\mathrm{F}$ & 4.35 & 2.02 & 4.35 & 2.02 & 0.519 & 0.131 & 1.04 & 0.499 & 3.96 & 0.0607 & 0.0274 \\
\hline
\end{tabular}

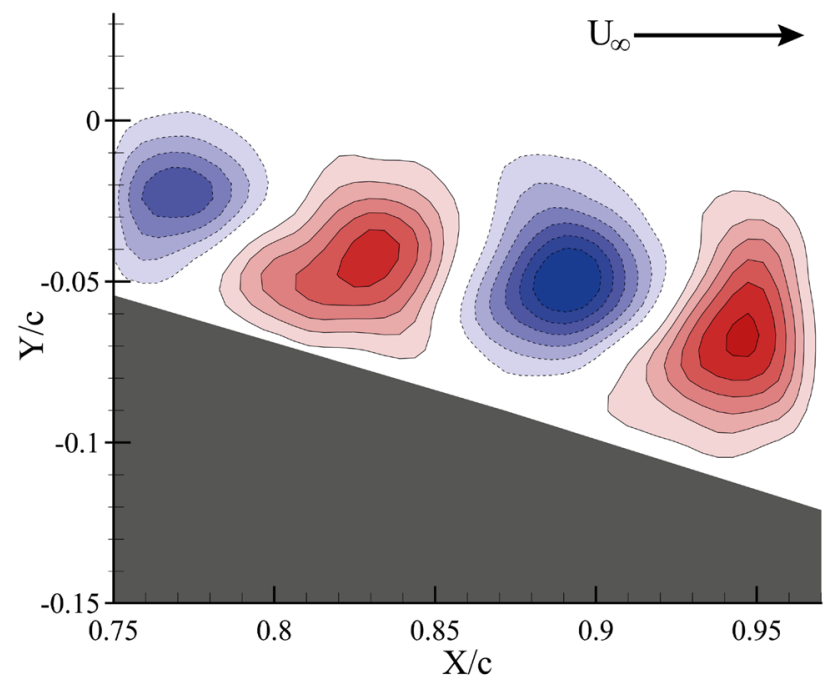

(a) Mode 4, corresponding to $f_{0}$

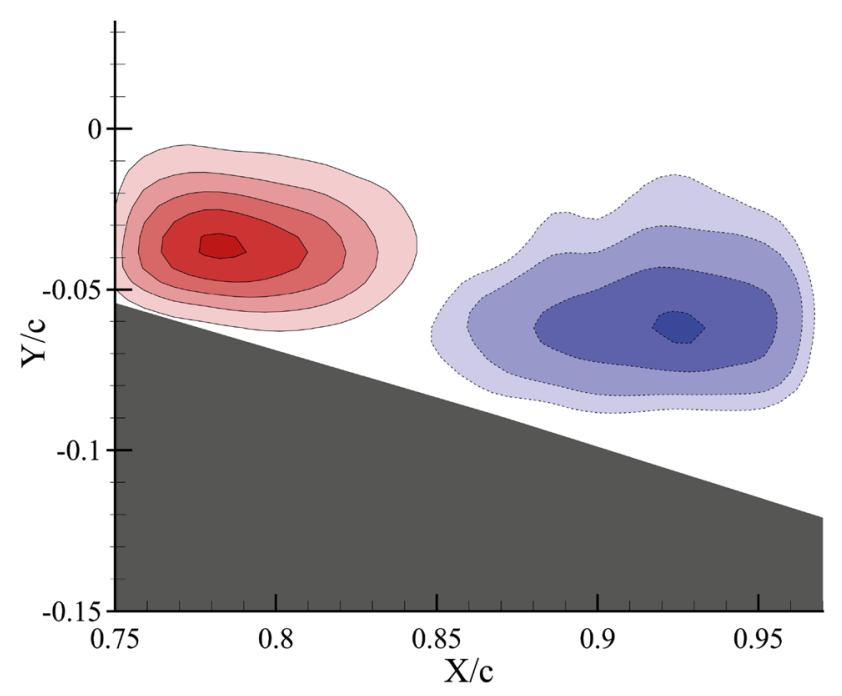

(b) Mode 2, corresponding to $f_{0-1 / 2}$

Fig. $4 v^{\prime}$ POD mode comparison for the case 150-10-F

averaged spectra showed no sensitivity in the result when compared to Fig. 5, and as such has not been presented here.

For the lowest Reynolds Number cases (100-10-P and 100-10-F), there was no dominating non-linear instabilities or vortex pairing (i.e. $f_{0-1 / 2}$ ) observed in the spectral analysis. The reason for this is thought to be because of the subcritical state of the shear-layer formation at the lower flow speed (Prasad and Williamson 1997; Rajagopalan and Antonia 2005).

When analysing the velocity probe data for the zero degree cases, it was seen that no obvious spectral peaks were present. The shear layer at these conditions is again expected to be in the sub-critical state as the adverse pressure gradient is weaker compared to the $10^{\circ}$ angle of attack situation (Huang and Lin 1995).

\subsection{Flaplet motion}

For the motion study, three neighbouring flaplets were analysed in order to distinguish between random motion patterns, representing turbulent structures, or spanwise coherent structures, such as the described rollers convecting along the flaplets. Each flap represents a one-sided clamped rectangular beam which oscillates in the first bending mode perpendicular to the long axis, at the natural frequency when being excited. The motion was recorded and analysed for $2.5 \mathrm{~s}$ leading to a total of 8000 frames. For further analysis, the flaplet motion has been classed into two different categories, synchronous in-phase (S) motion and antisynchronous (A-S) motion. The $\mathrm{S}$ motion is when all three flaplets are in the same phase, both for positive and negative deflections (see Fig. 6a for clarity). A-S motion is defined as when the flaplets have a phase delay of $\pi$ with respect to each other (see Fig. 6b). A small variance of the phase of $\pm 10^{\circ}$ is allowed when the time traces are investigated for such events. To investigate the probabilities of whether the flaplets have S or A-S motion the natural frequency of the flaplets, $f_{1}=107 \mathrm{~Hz}$, is used to calculate the maximum number of possible 'flap events' in the sampling time, which is 535 over the captured period. Events are only looked for when the centre flap is at its maximum positive or negative position.

When observing the $10^{\circ}$ cases in Table 5 , it can be seen that the $\mathrm{S}$ motion has a significantly higher probability than the A-S motion. This is proposed to be due to the presence of strong spanwise coherent shear-layer vortices. One might argue that the observed increase is due to the 


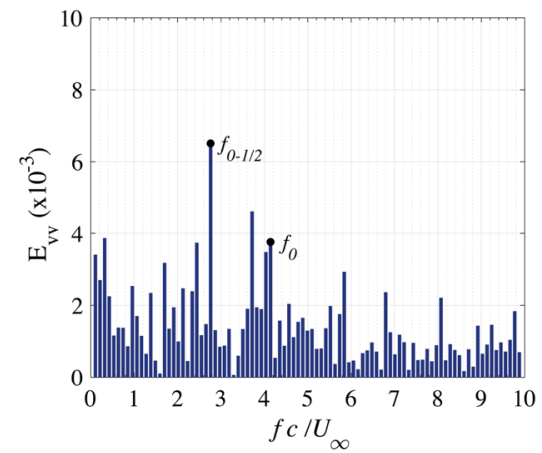

(a) 150-10-P; PIV Probe

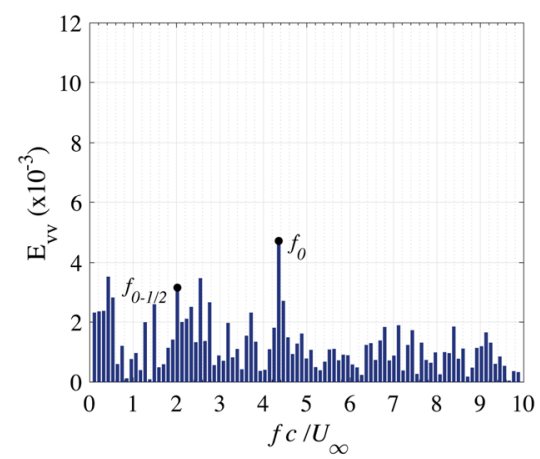

(d) 150-10-F; PIV Probe

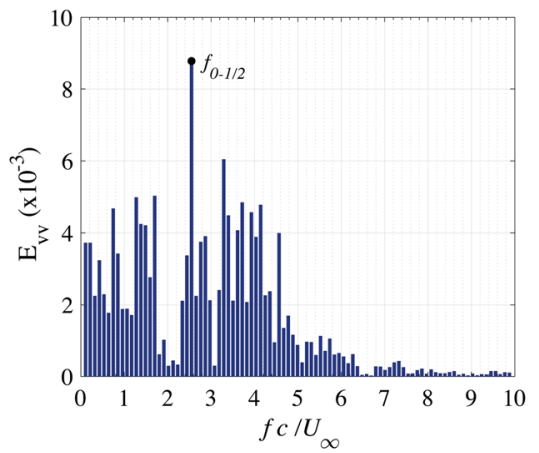

(b) $150-10-P ; 2^{\text {nd }} v^{\prime}$ POD mode

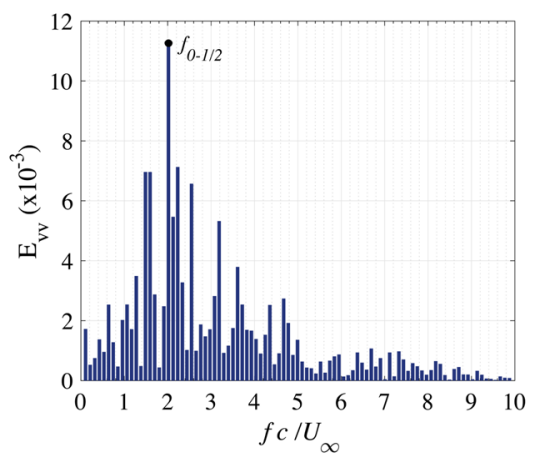

(e) $150-10-\mathrm{F} ; 2^{\text {nd }} v^{\prime}$ POD mode

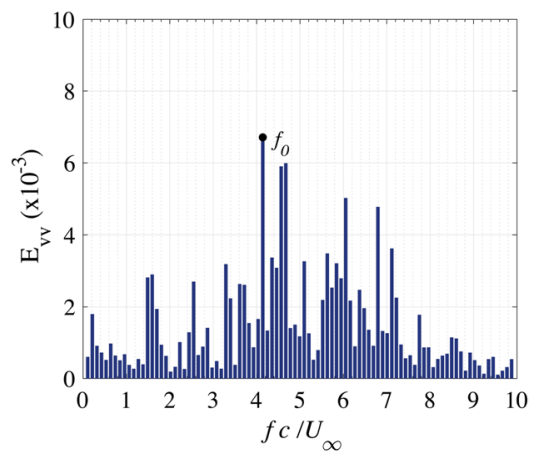

(c) $150-10-\mathrm{P} ; 4^{\text {th }} v^{\prime}$ POD mode

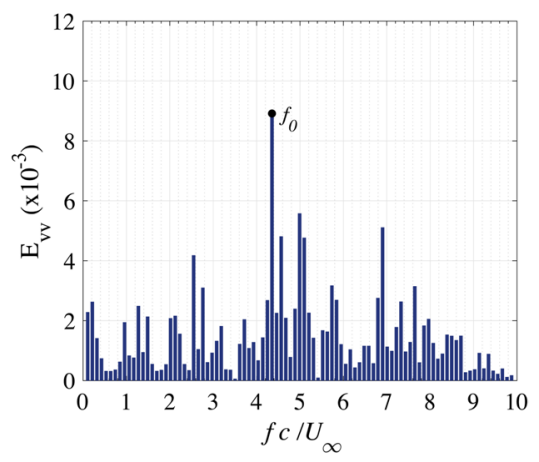

(f) $150-10-\mathrm{F} ; 4^{\text {th }} v^{\prime}$ POD mode

Fig. 5 Spectral analysis of the $v^{\prime}$ component from the velocity probe, 4th $v^{\prime}$ POD mode and 2nd $v^{\prime}$ POD mode at $R e_{\mathrm{c}}=150,000$ and at $\alpha=10^{\circ}$. a-c Baseline case. $\mathbf{d}-\mathbf{f}$ Flaplet case

spanwise mechanical coupling of the flaplets. However as can be seen in Fig. 7a, if one flaplet is excited then the neighbouring flaplets are coupled with a phase delay of $\pi$, as all flaplets are connected via a base layer. Figure $7 \mathrm{~b}$ shows that at the test case 150-10-F, the neighbouring flaplets are in phase with each other and have a strong correlation with each other (minimum 0.716). This indicated that a spanwise structure excited all three flaplets at the same time. An interesting observation is that the 100$10-\mathrm{F}$ case had a higher probability of $\mathrm{S}$ motion compared to $150-10-\mathrm{F}$. As the shear-layer fundamental frequency close to that of the natural frequency of the flaplets, it is hypothesised that the lock-in effect is more pronounced. A similar observation was made by Rosti et al. (2017) and hence a similar effect is proposed here. In comparison, for an angle of $0^{\circ}$, the flaplet motion is much more random. This is due to the weaker adverse pressure gradient on the upper side of wing, which reduces the mean shear in the inflection point of the boundary layer. Thus, the motion of the flaplets here is thought to be mainly due to small scale turbulent structures convected downstream and exciting the flaplets in a more random manner.

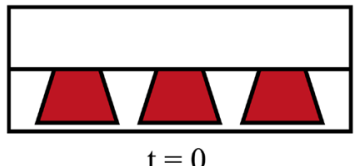

(a) Syncronous Motion
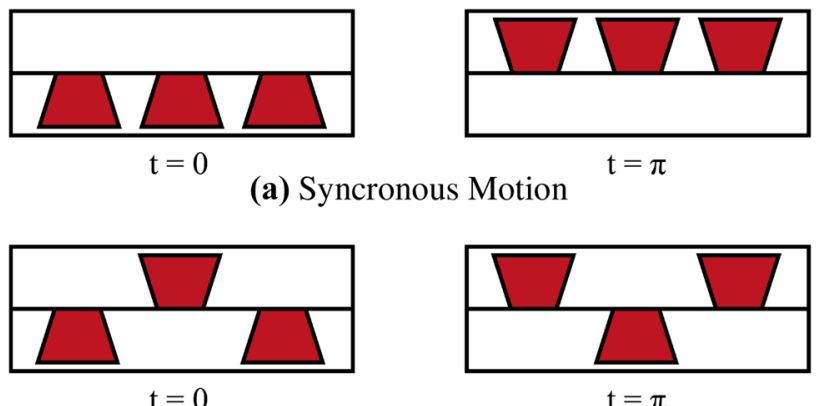

(b) Anti-syncronous Motion

Fig. 6 Sketch of the flaplets in either synchronous or anti-synchronous motion, looking from a downstream view of the trailing edge

\section{Conclusion}

Attaching thin flexible flaplets (oscillators) at the trailing edge of an aerofoil-free to oscillate in wall-normal direction-has been seen to have a profound upstream effect on the boundary layer at moderate angles of attack $\left(10^{\circ}\right)$; leading to a stabilisation of the fundamental mode growing in the shear layer on the suction side, along the second half of the wing. For the plain aerofoil, under the given flow conditions, 
Table 5 Flaplet motion synchronisation probabilities

\begin{tabular}{lll}
\hline Test case & $P(\mathrm{~S})$ & $P(\mathrm{~A}-\mathrm{S})$ \\
\hline $100-0-\mathrm{F}$ & 0.104 & 0.023 \\
$150-0-\mathrm{F}$ & 0.074 & 0.036 \\
$100-10-\mathrm{F}$ & 0.225 & 0.009 \\
$150-10-\mathrm{F}$ & 0.162 & 0.016 \\
\hline
\end{tabular}

spanwise coherent rollers are formed in the BL in the linear state and then further downstream the growth of non-linear instabilities leads to pairing of those rollers near the trailing edge. The pairing process is clearly suppressed when the flaplets are attached. They started to oscillate at their fundamental frequency with amplitudes of order of $1 \mathrm{~mm}$ $(0.5 \% \mathrm{c})$, excited by the convection of the rollers over the trailing edge. This observation leads to the conclusion that the stabilisation is due to a resonance or lock-in between the fundamental instability mode and the attached oscillators (flaplets), as this effect is most pronounced when the frequency of the fundamental shear-layer mode is closer to that of the natural frequency of the oscillators, given that the shear layer is already in its non-linear state. A previous study on bluff body wakes shows a similar stabilisation effect when flexible flaplets, being attached to the aft part, are getting into resonance with the vortex shedding cycle (Kunze and Brücker 2012). A further consequence of the lock-in stabilisation is the observed reduction in the boundary layer thickness. This is explained by the reduced probability of pairing events, which otherwise are responsible for larger wall-normal momentum exchange and a further thickening of the boundary layer. It is hypothesised therefore that the flaplets have a beneficial effect on the integral performance of the aerofoil such as reducing drag and increasing lift. This is supported by recent results of active trailing edges oscillators investigated at high frequency (Jodin et al. 2018). The present study also supports on physical means the earlier observation of the flaplets causing the dampening of trailing edge noise in the low-frequency range (Kamps et al. 2017). More recently, Talboys et al. (2018) have carried out an aeroacoustic study with the present configuration and indeed confirmed the reduction of acoustic noise in the low-to-medium frequency range $(100 \mathrm{~Hz}-1 \mathrm{kHz})$.

Compared to the very recent study on high-frequency active oscillating trailing edges (Jodin et al. 2018), the present method represents a charming alternative as a passive flow control technique, which does not require sophisticated and costly active manipulation techniques. As shown herein, the flaplets act as self-excited 'pacemakers' to stabilise the shear layer on the suction side and therefore it is reasonable to suggest that they also improve the aerodynamic performance of the aerofoil, besides helping to reduce trailing edge noise as already proven in Talboys et al. (2018). The natural frequency of these passive oscillators can be tailored for different flow situations either by using different shapes, intelligent materials with temperature- or pressure-dependent properties or by simply applying a deployment strategy where the length of the freely extending tips of the flaplets is varied by sliding the sheet along the trailing edge.
Fig. 7 Neighbouring flaplet motion

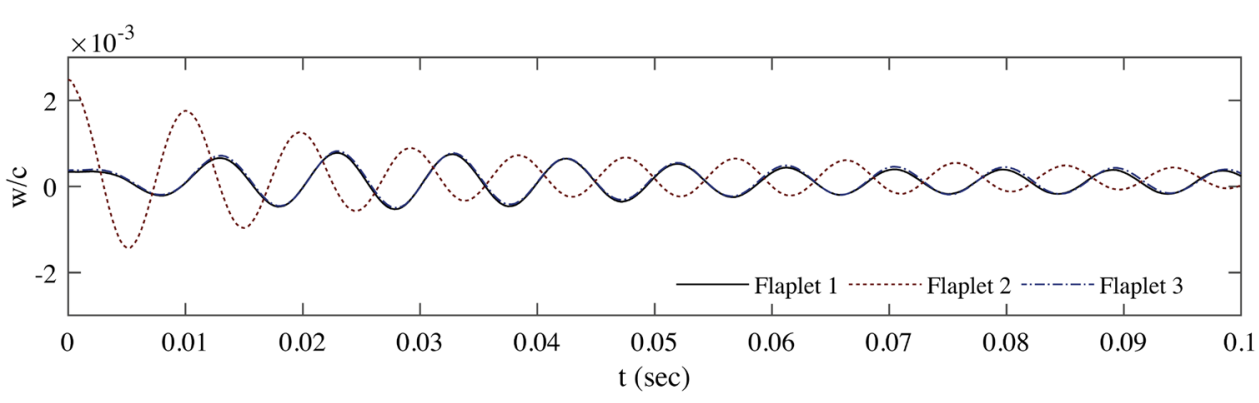

(a) Neighbouring flaplet response when only one flatlet, flaplet 2, is subjected to a deflection

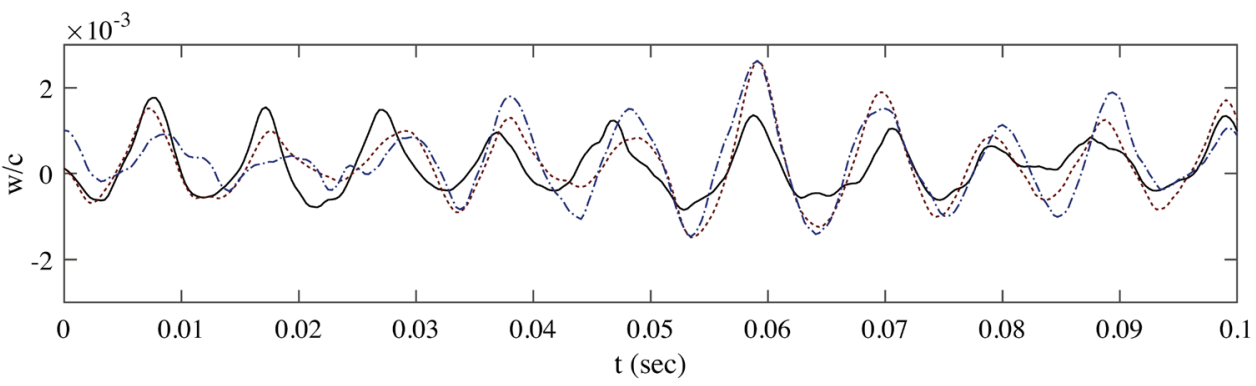

(b) Neighbouring flaplet motion measured at the test case 150-10-F 
Acknowledgements The position of Professor Christoph Brücker is co-funded by BAE SYSTEMS and the Royal Academy of Engineering (Research Chair No. RCSRF1617 \4\11), which is gratefully acknowledged.

Open Access This article is distributed under the terms of the Creative Commons Attribution 4.0 International License (http://creativeco mmons.org/licenses/by/4.0/), which permits unrestricted use, distribution, and reproduction in any medium, provided you give appropriate credit to the original author(s) and the source, provide a link to the Creative Commons license, and indicate if changes were made.

\section{References}

Boutilier MS, Yarusevych S (2012) Parametric study of separation and transition characteristics over an airfoil at low Reynolds numbers. Exp Fluids 52(6):1491-1506. https://doi.org/10.1007/s0034 8-012-1270-z

Brücker C, Weidner C (2014) Influence of self-adaptive hairy flaps on the stall delay of an airfoil in ramp-up motion. J Fluids Struct 47:31-40. https://doi.org/10.1016/j.jfluidstructs.2014.02.014

Carruthers AC, Thomas ALR, Taylor GK (2007) Automatic aeroelastic devices in the wings of a steppe eagle Aquila nipalensis. J Exp Biol 210(23):4136-4149. https://doi.org/10.1242/jeb.011197

Dong S, Karniadakis GE, Ekmekci A, Rockwell D (2006) A combined direct numerical simulation-particle image velocimetry study of the turbulent near wake. J Fluid Mech 569:185-207. https://doi. org/10.1017/S0022112006002606

Dovgal AV, Kozlov VV, Michalke A (1994) Laminar boundary layer separation: instability and associated phenomena. Prog Aerosp Sci 30(1):61-94. https://doi.org/10.1016/0376-0421(94)90003-5

Geyer TF, Sarradj E, Fritzsche C (2010) Measurement of the noise generation at the trailing edge of porous airfoils. Exp Fluids 48(2):291-308. https://doi.org/10.1007/s00348-009-0739-x

Geyer TF, Kamps L, Sarradj E, Brücker C (2017) Passive control of the vortex shedding noise of a cylinder at low Reynolds numbers using flexible flaps. In: 23rd AIAA/CEAS aeroacoustics conf, pp 1-11. https://doi.org/10.2514/6.2017-3015

Gruber M, Azarpeyvand M, Joseph P (2010) Airfoil trailing edge noise reduction by the introduction of sawtooth and slitted trailing edge geometries. In: Proc 20th int congr acoust ICA 10 August, pp 1-9

Herr M, Dobrzynski W (2005) Experimental investigations in lownoise trailing-edge design. AIAA J 46(6):1167-1175. https://doi. org/10.2514/1.11101

Ho CM, Huang LS (1982) Subharmonics and vortex merging in mixing layers. J Fluid Mech 119:443-473. https://doi.org/10.1017/ S0022112082001438

Howe MS (1991) Aerodynamic noise of a serrated trailing edge. J Fluids Struct 5(1):33-45. https://doi.org/10.1016/08899746(91)80010-B

Huang R, Lin C (1995) Vortex shedding and shear-layer instability of a cantilever wing at low Reynolds numbers. In: 33rd aerosp sci meet exhib, vol 33, no 8, pp 1398-1403. https://doi.org/10.2514/3.12561

Jaworski JW, Peake N (2013) Aerodynamic noise from a poroelastic edge with implications for the silent flight of owls. J Fluid Mech 723:456-479. https://doi.org/10.1017/jfm.2013.139

Jodin G, Rouchon JF, Scheller J, Triantafyllou M (2018) Electroactive morphing vibrating trailing edge of a cambered wing: PIV, turbulence manipulation and velocity effects. In: IUTAM symp. crit. flow dyn. involv. moving/deformable struct. with des. appl., Santorini

Kamps L, Geyer TF, Sarradj E, Brücker C (2016) Vortex shedding noise of a cylinder with hairy flaps. J Sound Vib 388:69-84. https ://doi.org/10.1016/j.jsv.2016.10.039
Kamps L, Brücker C, Geyer TF, Sarradj E (2017) Airfoil self noise reduction at low Reynolds numbers using a passive flexible trailing edge. In: 23rd AIAA/CEAS aeroacoustics conf., June. American Institute of Aeronautics and Astronautics, Reston, pp 1-10. https://doi.org/10.2514/6.2017-3496

Kunze S, Brücker C (2012) Control of vortex shedding on a circular cylinder using self-adaptive hairy-flaps. Comptes Rendus Mec 340(1-2):41-56. https://doi.org/10.1016/j.crme.2011.11.009

Meyer KE, Cavar D, Pedersen JM (2007) POD as tool for comparison of PIV and LES data. In: 7th int symp part image velocim, pp $1-12$

Miklosovic DS, Murray MM, Howle LE, Fish FE (2004) Leadingedge tubercles delay stall on humpback whale (Megaptera novaeangliae) flippers. Phys Fluids 16(5):L39-L42. https://doi. org/10.1063/1.1688341

Osterberg N, Albertani R (2017) Investigation of self-deploying high-lift effectors applied to membrane wings. Aeronaut J 121(1239):660-679. https://doi.org/10.1017/aer.2017.10

Perret L (2009) PIV investigation of the shear layer vortices in the near wake of a circular cylinder. Exp Fluids 47(4-5):789-800. https:// doi.org/10.1007/s00348-009-0665-y

Ponitz B, Schmitz A, Fischer D, Bleckmann H, Brücker C (2014) Diving-flight aerodynamics of a peregrine falcon (Falco peregrinus). PLoS One 9(2):e86506. https://doi.org/10.1371/journ al.pone.0086506

Prasad A, Williamson CHK (1997) The instability of the shear layer separating from a bluff body. J Fluid Mech 333:S0022112096004326. https://doi.org/10.1017/S002211209 6004326

Rajagopalan S, Antonia RA (2005) Flow around a circular cylinderstructure of the near wake shear layer. Exp Fluids 38(4):393-402. https://doi.org/10.1007/s00348-004-0913-0

Rodríguez I, Lehmkuhl O, Borrell R, Oliva A (2013) Direct numerical simulation of a NACA0012 in full stall. Int J Heat Fluid Flow 43:194-203. https://doi.org/10.1016/j.ijheatfluidflow.2013.05.002

Rosti ME, Kamps L, Brücker C, Omidyeganeh M, Pinelli A (2017) The PELskin project-part V: towards the control of the flow around aerofoils at high angle of attack using a self-activated deployable flap. Meccanica 52(8):1811-1824. https://doi.org/10.1007/s1101 2-016-0524-x

Schlüter JU (2010) Lift enhancement at low Reynolds numbers using self-activated movable flaps. J Aircr 47(1):348-351. https://doi. org/10.2514/1.46425

Semeraro O, Bellani G, Lundell F (2012) Analysis of time-resolved PIV measurements of a confined turbulent jet using POD and Koopman modes. Exp Fluids 53(5):1203-1220. https://doi. org/10.1007/s00348-012-1354-9

Stanek FJ (1965) Free and forced vibrations of cantilever beams with viscous damping. Tech. Rep, June. National Aeronautics and Space Administration, Washington DC

Talboys E, Geyer TF, Brücker C (2018) The aerodynamic and aeroacoustic effect of passive high frequency oscillating trailing edge flaplets. In: IUTAM symp. crit. flow dyn. involv. moving/deformable struct. with des. appl., Santorini

Thomareis N, Papadakis G (2017) Effect of trailing edge shape on the separated flow characteristics around an airfoil at low Reynolds number: a numerical study. Phys Fluids 29(1):014101. https://doi. org/10.1063/1.4973811

Yarusevych S, Sullivan PE, Kawall JG (2009) On vortex shedding from an airfoil in low-Reynolds-number flows. J Fluid Mech 632:245. https://doi.org/10.1017/S0022112009007058

Publisher's Note Springer Nature remains neutral with regard to jurisdictional claims in published maps and institutional affiliations. 